\title{
Immunotherapy-Related Adverse Effects When Treating Cancer \#375
}

\author{
Christina Matts, MD and Anna Beck, MD
}

\section{Background}

C HECKPOINT IMMUNOTHERAPY is a rapidly evolving treatment paradigm for solid organ cancers. ${ }^{1}$ These medications are often antibodies that target key regulators of the immune system to unleash an immune system attack on cancer cells. Examples include cytotoxic T-lymphocyteassociated antigen 4 (CTLA-4) inhibitors (e.g., ipilimumab) or programmed death receptor-1 (PD-1) inhibitors (e.g., pembrolizumab and nivolumab). See Fast Fact \#277 for more information. Although heightened immune response against the tumor cells is intended, healthy tissues can also be attacked leading to unintended inflammation of almost any organ system. This has led to a unique set of immune-related adverse events (IRAEs). Given the expanding use of checkpoint immunotherapy, clinical awareness of IRAEs is important among generalist and palliative care clinicians.

\section{Immune-Related Adverse Events}

Early recognition of IRAEs is paramount. The National Cancer Institute along with the National Institute of Health published definitions for adverse events. Grade 1-2 IRAEs are considered mild to moderate in severity. Grade 3 is more severe and often requires hospitalization. Grade 4 is a lifethreatening IRAE and grade 5 represents a fatal IRAE. ${ }^{2,3}$ Some IRAEs are misattributed to disease progression by clinicians unfamiliar with immunotherapy. Therefore, it is important to inform the patient's primary oncologist whenever an IRAE is suspected or identified. Although corticosteroids are common treatments that help manage IRAEs, it is strongly advised that clinicians confer with the patient's oncologist before initiating them as they may temper the oncological response of immunotherapy.
- Cutaneous: Pruritus, vitiligo, and an erythematous maculopapular rash involving the trunk and extremities are the most common IRAEs. ${ }^{2,4}$ They typically manifest two to three weeks after treatment initiation, which is much earlier than other IRAEs. Cutaneous toxicities are more common with CTLA-4 inhibitors, except for vitiligo that occurs more often with PD-1 inhibitors. ${ }^{5}$ The development of a rash, particularly vitiligo, may be a favorable prognostic sign corresponding with improved progression-free and overall survival. ${ }^{4}$ In rare cases, severe cutaneous reactions such as StevensJohnson syndrome or toxic epidermal necrolysis occur, which usually require urgent ICU admission along with a dermatology and oncology consultation.

Management: Grade 1 toxicities are usually treated with topical emollients, urea-containing creams, oral antihistamines, and topical steroids. ${ }^{4}$ For grade 2 toxicities, immunotherapy is often held until the reaction improves to grade 1 . Oral prednisone at $0.5-1 \mathrm{mg} /(\mathrm{kg}$ per day) can be considered with a taper for four weeks. Grades $\geq 3$ toxicity require intravenous (IV) methylprednisolone (or equivalent) at $1-2 \mathrm{mg} /(\mathrm{kg}$ per day) with a taper for 4 weeks. For grade 4 toxicities, for example, those with bullous lesions and/or mucous membrane involvement, immunotherapy is typically discontinued permanently.

- Gastrointestinal: Diarrhea, colitis, and hepatitis are quite common. In fact, diarrhea occurs in $>50 \%$ of patients treated with CTLA- 4 inhibitors. ${ }^{2}$ Most GI IRAEs begin 5-10 weeks after immunotherapy exposure, except for hepatitis that often begins 6-16 weeks after treatment exposure. ${ }^{2,4}$ Colitis often presents as abdominal cramping or bloating, blood or mucous in the stool, and fever. Patients with hepatitis often have an asymptomatic elevation in their liver function tests but

Fast Facts and Concepts are edited by Sean Marks, MD (Medical College of Wisconsin), and associate editor Drew A. Rosielle, MD (University of Minnesota Medical School), with the generous support of a volunteer peer-review editorial board, and are made available online by the Palliative Care Network of Wisconsin (PCNOW); the authors of each individual Fast Fact are solely responsible for that Fast Fact's content. The full set of Fast Facts is available at PCNOW with contact information, and how to reference Fast Facts.

Version History: originally edited by Sean Marks; first electronically published in March 2019.

Copyright: All Fast Facts and Concepts are published under a Creative Commons Attribution-NonCommercial 4.0 International Copyright (http://creativecommons.org/licenses/by-nc/4.0). Fast Facts can only be copied and distributed for noncommercial, educational purposes. If you adapt or distribute a Fast Fact, let us know!

Disclaimer: Fast Facts and Concepts provide educational information for health care professionals. This information is not medical advice. Fast Facts are not continually updated, and new safety information may emerge after a Fast Fact is published. Health care providers should always exercise their own independent clinical judgment and consult other relevant and up-to-date experts and resources. Some Fast Facts cite the use of a product in a dosage, for an indication, or in a manner other than that recommended in the product labeling. Accordingly, the official prescribing information should be consulted before any such product is used. 
then eventually develop nausea, vomiting, jaundice, and abdominal pain. Iatrogenic deaths from CTLA-4 inhibitors are usually related to colonic perforation from unrecognized colitis. ${ }^{5}$

Management: Grade 1 toxicities often require observation and supportive care. See Fast Fact \#96 for management of diarrhea. For grade 2, immunotherapy is held until toxicities improve to grade 1 or resolve. Four weeks of oral prednisone could be considered. Grade $\geq 3$ toxicities are treated with IV methylprednisolone (at similar dose as cutaneous reactions) and usually require hospitalization and permanent discontinuation of the immunotherapy. Infliximab can be considered for most GI IRAEs refractory to IV corticosteroids, except for hepatitis, as it can worsen hepatotoxicity. ${ }^{6}$

- Pulmonary: Although the incidence of pneumonitis is only $2-6 \%$, with a slight predominance in those treated with PD-1 inhibitors, it can be fatal. ${ }^{4}$ IRAE-induced pneumonitis may present initially with only a dry cough or mild dyspnea, hence high clinical suspicion is needed to make the diagnosis. Onset time varies but is usually between 8 and 14 weeks. ${ }^{6}$

Management: Recommendations mirror those for GI IRAEs except that empiric antibiotics should be considered for grade $\geq 3$ toxicities. $^{6}$

- Endocrine: Clinically significant immune-related endocrinopathies occur in about $10 \%{ }^{4}$ Hypothyroidism, hyperthyroidism, adrenal insufficiency, and type $1 \mathrm{di}-$ abetes mellitus are most common and can have significant clinical consequences if not recognized.

Management: An endocrinology consultation is usually recommended since lifelong hormone replacement therapy may be required. ${ }^{6,7}$ Once stabilized, most can resume immunotherapy.

- Rare IRAEs: There have been case reports of fatal myocarditis and neurological toxicities such as myasthenia gravis and acute inflammatory neuropathies (often labeled as Gullian-Barre syndrome). Immunotherapy is permanently discontinued for grade $\geq 3$ cardiac or neurological IRAEs. ${ }^{8}$ Intravenous immunoglobulin and/or plasmapheresis may be required for IRAEs refractory to IV corticosteroids. ${ }^{8}$

- Pseudoprogression: Although not an IRAE, it represents a unique response to immunotherapy and involves immune cell tumor infiltration. This can cause the appearance of tumor growth or new lesions on radiological imaging. Pseudoprogression usually resolves within 612 weeks, but it can be difficult to discern from true cancer progression. It is relatively uncommon, occurring in just $2-14 \%{ }^{9}$ Still, its diagnosis requires considerable clinical judgment and can have important prognostic implications. In general, if patients are doing well clinically, immunotherapy is continued, and lesions are reassessed through a short-interval radiological study. If the patient is clinically deteriorating, the imaging findings usually represent true disease progression.

\section{References}

1. Postow MA, Sidlow R, Hellmann MD: Immune-related adverse events associated with immune checkpoint blockade. N Engl J Med 2018;378158-168.

2. Kumar V, Chaudhary N, Garg M, et al.: Corrigendum: Current diagnosis and management of immune related adverse events (IrAEs) induced by immune checkpoint inhibitor therapy. Front Pharmacol 2017;8:311.

3. Abdel-Wahab N, Alshawa A, Suarez-Almazor ME: Adverse events in cancer immunotherapy. Adv Exp Med Biol 2017; 995:155-174.

4. Brahmer JR, Lacchetti C, Thompson JA: Management of immune-related adverse events in patients treated with immune checkpoint inhibitor therapy: American Society of Clinical Oncology Clinical Practice Guideline summary. J Oncol Pract 2018; DOI: 10.1200/jop.18.00005.

5. Khoja L, Day D, Wei-Wu Chen T, et al.: Tumour- and classSpecific patterns of immune-Related adverse events of immune checkpoint inhibitors: A systematic review. Ann Oncol 2017;28:2377-2385.

6. Friedman CF, Proverbs-Singh TA, Postow MA: Treatment of immune related adverse effects of immune check-point inhibitors. JAMA Oncol 2016;2:1346-1353.

7. Sznol M, Postow MA, Davies MJ, et al.: Endocrine-related adverse events associated with immune checkpoint blockade and expert insights on their management. Cancer Treat Rev 2017;58:70-76.

8. Kao JC, Liao B, Markovic SN, et al.: Neurological complications associated with anti-programmed death 1 (PD-1) antibodies. JAMA Neurol 2017;74:1216.

9. Wang GX, Kurra V, Gainor JF, et al.: Immune checkpoint inhibitor cancer therapy: Spectrum of imaging findings. Radiographics 2017;37:2132-2144.

Address correspondence to: Anna Beck, MD

Clinic 2B, Supportive Oncology and Survivorship (SOS) Huntsman Cancer Hospital University of Utah 1950 Circle of Hope Salt Lake City, UT 84112

E-mail: anna.beck@hci.utah.edu 